\title{
A Linguistic Decision Model for Promotion Mix Management Based on Genetic Algorithms
}

\author{
Xihui Li Zheng Pei Hua Zhao \\ School of Mathematics \& Computer Science, Xihua University, Chengdu 610039, China
}

\begin{abstract}
As we know, promotion activities are very important marketing tools. As a result of this fact, the selection of activities is an important decision. In this paper, from the viewpoint of satisfaction degree and total expense amount, we design a reasonable evaluation function, and use genetic algorithms to search for a desirable solution in promotion activities, the conclusion shows that our method could both come to certain the objectives of company and minimize the investment amount of company.
\end{abstract}

Keywords: Linguistic decision model, The $L O W A$ operator, Genetic algorithms, Evaluation function

\section{Introduction}

Promotion may be considered as the process through which an organization communicates with, and influences. Its target market segments with the goal of helping to position its products or services in their desired locations and generating the desired response from the segments.

The promotion mix selection constitutes an important decision, since the customer's behavior is clearly determined by it. With this decision, the firm must select the marketing tools that accomplished its short-term communication objectives.

The aim of this paper is to attempt to devise a decision model for promotion mix management in conditions of uncertainty, supplying a linguistic decision model for evaluating the satisfaction of the objectives by the potential solutions, such that it will both accomplish the communication objectives of the company and minimize the invested amount[1]. Therefor, we consider the two decision factors and search for a correspondingly better solution based on genetic algorithms. In Section 2, the promotion mix management problem and genetic algorithms are briefly reviewed. In section 3, another method based on $G A$ to search for a better solution model is presented. The conclusion is section 4 .

\section{Linguistic promotion mix management problem}

As we have mentioned, promotion activities may be considered as the process through which a company could deserve a satisfied objective after investing a small quantity of money. For example, a possible solution in promotion mix management model $S=$ $\left(S_{1}, S_{2}, \cdots, S_{n}\right)$ must satisfy $\sum_{h=1}^{n} S_{h} \leq T$, where $S_{h}$ represents the investment amount level on the $h$ th promotion tool, with $H_{h}$ being the number of insertions made for the tool, with $C_{H_{h}}^{h}=S_{h}$ [1]. In application, we have to synthetically evaluate this possible solution according to some principles, such as the objective of promotion model and the total quantity of investment and so on.

\subsection{Objective of promotion}

In the promotion mix management problem, we could select different activity tools like television advertising, discount and newspaper article,etc. Through using these tools properly, the promotion activity could come to a desirable result. But we know that lots of knowledge is given in the form of fuzzy information. Let us take the objectives of promotion for example, we could deserve different solutions called satisfaction degrees, which are denoted by fuzzy linguistic information such as high moderate or low.

Usually, we use the specification of the kind of label set to represent relative semantic information in the application. Then, let $S=\left\{s_{i}\right\}, i \in H=$ $\{0, \cdots, T\}$ be a finite and totally ordered term set on $[0,1]$ in the usual sense $[2,9]$. Any label, $s_{i}$, represents a possible value for a linguistic variable, that is a vague property or constraint on $[0,1]$. We 
consider a term set, $S$, with its semantics given by linear triangular membership functions. Moreover, it must have the following characteristics:

(1) There is a negation operator: $\operatorname{Neg}\left(s_{i}\right)=s_{j}$ such that $j=T-i$;

(2) The set is ordered: $s_{i} \geqslant s_{j}$ if $i \geqslant j$;

(3) There are a maximization and a minimization operator:

$$
\begin{aligned}
& \max \left(s_{i}, s_{j}\right)=s_{i} \text { if } s_{i} \geqslant s_{j} \\
& \min \left(s_{i}, s_{j}\right)=s_{i} \text { if } s_{i} \leqslant s_{j} .
\end{aligned}
$$

A wide study on the choice of a linguistic term set can be found in [4]. In this paper, we have chosen a set of nine linguistic labels as shown in Fig 1[1].

\section{Nine Term Set}

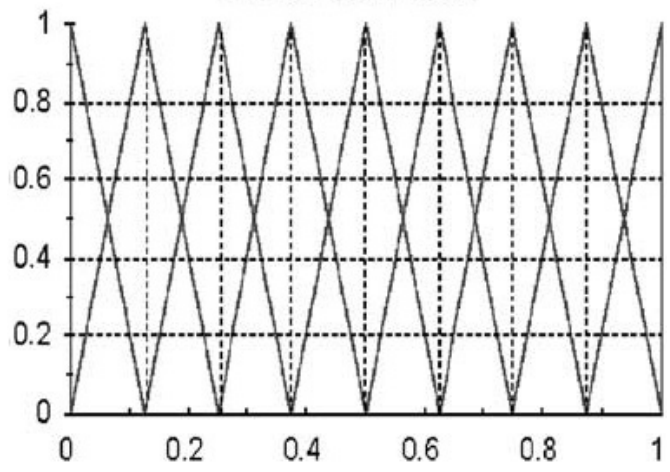

Fig. 1: Linguistic nine term set

The 3-tuples associated are:

\begin{tabular}{lll}
\hline E & Essential $\left(s_{9}\right)$ & $(0.875,1,1)$ \\
VH & Very high $\left(s_{8}\right)$ & $(0.75,0.875,1)$ \\
FH & Fairly high $\left(s_{7}\right)$ & $(0.625,0.75,0.875)$ \\
H & $\operatorname{High}\left(s_{6}\right)$ & $(0.5,0.625,0.75)$ \\
M & Moderate $\left(s_{5}\right)$ & $(0.375,0.5,0.625)$ \\
L & Low $\left(s_{4}\right)$ & $(0.25,0.375,0.5)$ \\
FL & Fairly low $\left(s_{3}\right)$ & $(0.125,0.25,0.375)$ \\
VL & Very low $\left(s_{2}\right)$ & $(0,0.125,0.25)$ \\
$\mathrm{U}$ & Unnecessary $\left(s_{1}\right)$ & $(0,0,0.125)$ \\
\hline
\end{tabular}

From the table above, we can see nine language labels which stand for different linguistic information. In the promotion mix management model, these labels could denote certain linguistic evaluation information. A company transmits the information to its target market with the goal of helping to position its products or services and generating the desired response from the customers. See the following table.

Table 1:

\begin{tabular}{lll}
\hline TV advertising & One insertion & Two insertion \\
\hline Cost & 3000 & 5000 \\
Knowledge & Very High & Essential \\
Recognition & High & Very High \\
Acquisition & Moderate & Moderate \\
Preference & Very Low & Low \\
Loyalty & Moderate & High \\
\hline
\end{tabular}

We find that the activity of $T V$ advertising has two kinds of investment level. At the same time, linguistic evaluation information reflects on five different objectives (cost, knowledge, recognition, acquisition, preference). Similarly, other activities have relative information. In the promotion mix management model, we must consider the final satisfaction degree according to each tool's linguistic evaluation information. Therefor, the linguistic aggregation operators are needed to appropriately combine the information. In this paper, the label set of nine terms is denoted as

$\mathrm{S}=\left\{S_{9}=\right.$ Essential,$S_{8}=$ Veryhigh,$S_{7}=$ Fairlyhigh, $S_{6}=$ High,$S_{5}=$ Moderate, $S_{4}=$ Low,$S_{3}=$ Fairly low,$S_{2}=$ Verylow,$S_{1}=$ Unnecessary $\}$

Definition 1 An linguistic $O W A$ operator is defined as follows:

$\operatorname{LOW} A_{w}\left(s_{1}, s_{2}, \cdots, s_{n}\right)=$ $w_{1} \odot s_{\beta_{1}} \oplus w_{2} \odot s_{\beta_{2}} \oplus \cdots \oplus w_{n} \odot s_{\beta_{n}}$

where $s_{i} \oplus s_{j}=s_{i+j}, \quad \lambda \odot s_{i}=s_{\lambda i}$. $W^{T}=\left(w_{1}, w_{2}, \cdots, w_{n}\right)$ is weighting vector, such that $w_{i} \in[0,1]$ and $\sum_{i} w_{i}=1 . s_{\beta j}$ is the $j$ th largest $s_{i}$. Besides, the weighting vectors $w_{i}$ can be computed by linguistic quantifier $Q$ defined by Yager, are given by this expression[2]:

$w_{j}=Q(j / n)-Q((j-1) / n), j=1, \cdots, k$.

So if we have a set of linguistic labels, we could get the evaluation result of solution in the goals of the promotion and the result is denoted as $s_{\alpha}$. If $s_{\alpha} \in S$, then we call sa an original linguistic term, otherwise, we call $s_{\alpha}$ virtual linguistic term [?]. We could evaluate the result according to the subscript value of $S$.

\subsection{Genetic algorithms}

Genetic algorithms $(G A)$ are search algorithm that use operations found in natural genetics to guide the trek through a search space. $G A$ are theoretically and empirically proven to provide robust search capabilities in complex spaces,offering 
a valid approach to problems requiring efficient and effective search[6]-[8]. A $G A$ starts with a population of randomly generated chromosomes, and advances towards better chromosomes by applying genetic operators modelled on the genetic processes occurring in nature. The population undergoes evolution in a form of natural selection. During successive iteration, called generations, chromosomes in the population are rated for their adaptation as solutions, and on the basis of these evaluation, a new population of chromosomes is formed using a selection mechanism and specific genetic operators such as crossover and mutation. And evaluation or fitness function must be devised for each problem to be solved.Given a particular chromosome, a possible solution, the fitness function returns a single numerical value, which is supposed to be proportional to the utility or adaption of the solution represented by that chromosome[5]. GAs play a significant role as search techniques for handing complex spaces in many fields, particularly in management problems[10]-[14].

When we use GAs to solve a problem, we must take into account the following components:

1. Randomly create an initial population.

2. Design a proper evaluation function named fitness degree function.

3. The choice of genetic operator concluding selection, crossover and mutation.

4. Design the value of parameters used in $G A s$, such as the number of generation, the probability of crossover and mutation, etc.

We utilize genetic algorithms to optimize the promotion mix model, then company could invest a small quantity of money and deserve a desired result.

\section{Finding a better solution model based on another $G A s$ theory}

In this section, a usual linguistic decision model will be given. There are eight tools (Television advertising, Radio advertising, Newspaper advertising, Salesman, Discount, Prize, Free sample, Newspaper article) which could be selected to invest money and there are five different evaluation objectives (Knowledge, Recognition, Acquisition, Preference, Loyalty). The set $S=(3000,2000,0,2000,0,0,3000,0)$ is a possible solution in this decision model. A abstract model is described in the following table.

\begin{tabular}{lllllll}
\hline Tools & Investment & $\mathrm{O}_{1}$ & $\mathrm{O}_{2}$ & $\mathrm{O}_{3}$ & $\mathrm{O}_{4}$ & $O_{5}$ \\
\hline$T_{1}$ & 3000 & $\mathrm{VH}$ & $\mathrm{H}$ & $\mathrm{M}$ & $\mathrm{VL}$ & $\mathrm{M}$ \\
$T_{2}$ & 2000 & $\mathrm{VL}$ & $\mathrm{L}$ & $\mathrm{M}$ & $\mathrm{VL}$ & $\mathrm{M}$ \\
$T_{3}$ & 0 & - & - & - & - & - \\
$T_{4}$ & 2000 & $\mathrm{~L}$ & $\mathrm{M}$ & $\mathrm{H}$ & $\mathrm{H}$ & $\mathrm{FH}$ \\
$T_{5}$ & 0 & - & - & - & - & - \\
$T_{6}$ & 0 & - & - & - & - & - \\
$T_{7}$ & 3000 & $\mathrm{VL}$ & $\mathrm{VL}$ & $\mathrm{FH}$ & $\mathrm{H}$ & $\mathrm{FL}$ \\
$T_{8}$ & 0 & - & - & - & - & - \\
\hline
\end{tabular}

According to the knowledge which have been mentioned in section 2, we could compute the possible solution's total expense amount. It is

$\sum_{h=1}^{8} S_{h}=T_{s}=3000+2000+0+2000+$ $0+0+3000+0=10000$.

As to the satisfaction degree, we need to use $L O W A$ operator which have been defined in section 2. The first step, we aggregate linguistic evaluation value on each objective. Take the objective $1\left(O_{1}\right)$ for example. The label set is $\left(s_{8}, s_{2}, s_{4}, s_{2}\right)$ and the weighting vector is $(0$, $0.4,0.5,0.1)$. Using the aggregation operator, the evaluation result of $\left(O_{1}\right)$ is $s_{3}$. Similarly, other objective's evaluation results are $s_{4.3}, s_{5.7}$, $s_{4.4}, s_{5.8}$. The second step, we aggregate the five objectives'evaluation results $S_{x}$. The weighting vector is $(0,0.2,0.4,0.4,0)$. Finally, the collective satisfaction degree is $s_{4.9}$, that is, its linguistic evaluation result greatly approaches Moderate.

Now we optimize the promotion decision model based on another GAs theory. The detailed process is in the following section.

\subsection{Initial gene poll}

To begin with, we designate the number of chromosome in each generation(popsize) is 10 and the number of generation is 100 . Because each tool has its own number of insertion, we randomly generate a set about insertion such as $H=\left(\begin{array}{llllll}0 & 1 & 0 & 1 & 2 & 0\end{array}\right.$ $10)$. The elements in this set could be changed into a set whose elements are investment amount on each tool. This operation aims at easily generating a group of possible solutions in the initial population. For chromosomes'fitness degree in initial population are much better, there are some limits on the elements in this group of sets. Firstly, every element must be probable insertion value in the original information. Secondly, the element sequence stands for a probable solution in the decision model, so its total expense amount should verifies that $T_{s} \leq T$, where $\sum_{h=1}^{k} S_{h} \leq T_{s}$ and 
$T_{s}=10000$. Besides, its satisfaction degree is not lower the one which has been deserved in the first possible solution. Any solution which can not satisfy the limits above, we should discard it and generate the new possible solution again. At last, we could generate the initial population whose chromosomes show probable solutions. Next, we use the $G A s$ operators to generate the next generation.

\subsection{Evaluation function}

In the initial gene poll, we need a evaluation function to ascertain each chromosome's fitness value. We all know those chromosomes which have much higher fitness value can easily live and have more chance to be selected in the next generation. In promotion mix management problem, we must consider two factors to design the evaluation function. One is the total investment amount and the other is the final objective satisfaction degree of decision model. The following is the fitness function.

$$
f(i)=\left\{\left(10000-\sum_{j=1}^{n} s_{i}(j)\right)+\gamma \times \sum_{k=1}^{m} w_{i}(k) t_{i}(k)\right\}
$$

Where $i$ is $i$ th individual and $1 \leq i \leq$ popsize, $n$ is the number of tools, $m$ is the number of objectives, $\gamma$ is a balance parameter. Besides

$$
t_{i}(k)=\sum_{k, h=1}^{l} w_{h}^{k} \delta\left(b_{h}^{k}\right)
$$

Where $\delta\left(b_{h}\right)=h$ and $l$ is the number of tools which have evaluation information.

\subsection{Selection}

According to the fitness function we have proposed above, we could get a sequence of value. Then we sort the sequence value from high to low. Now we design three parameters $r 1, r 2$. The first parameters is reservation gene, the second is reproduction gene and the third is random gene. Using the two parameters, we could generate the next generation. The reservation gene is used to decide how many individuals are reserved. The number of this part is $N=r 1 \times N$ where $N$ is the number of individuals in the previous generation. The reproduction gene is used to decide how many individuals operate the process of crossover and mutation. The number of this part is $M=r 2 \times N$. Hence, The individual number in the new population are composed of $N$ and $M$. In this paper, we let $r 1=\frac{1}{4} \times N$ and $r 2=\frac{5}{8} \times N$. As to the spare chromosomes, which have much low fitness degree in this population, we discard them.

\subsection{Crossover}

According to the reproduction gene, a part of individuals are regarded as parents and carry through crossover operation. In this paper, we choose the simple crossover method called one-point crossover, which is the most basic crossover mode proposed by Holland. For example, the parents are S1 and S2 and their composition could be

$S 1=\left(a_{11}, a_{12}, \cdots, a_{1 l_{1}}, a_{1 l_{2}}, \cdots, a_{1 L}\right)$,

$S 2=\left(b_{11}, b_{12}, \cdots, b_{1 l_{1}}, b_{1 l_{2}}, \cdots, b_{1 L}\right)$.

Then we choose a random point $x$. And it satisfies $x \epsilon\{1,2, \cdots, L-1\}$, supposing that $l_{1} \leq x \leq l_{2}$. Then we make the right of crossover point change and generate the new offspring. After this operation, they become

$S 1^{\prime}=\left(a_{11}, a_{12}, \cdots, a_{1 l_{1}}, b_{1 l_{2}}, \cdots, b_{1 L}\right)$,

$S 2^{\prime}=\left(b_{11}, b_{12}, \cdots, b_{1 l_{1}}, a_{1 l_{2}}, \cdots, a_{1 L}\right)$.

For example, the obtained parents could be

$\mathrm{S} 1=(0,2000,0,4000,1000,0,3000,0)$, $\mathrm{S} 2=(3000,0,0,2000,1800,0,3000,0)$.

Supposing the crossover point is the last four elements, so they become

$\mathrm{S} 1^{\prime}=(0,2000,0,2000,1800,0,3000,0)$, $\mathrm{S} 2^{\prime}=(3000,0,0,4000,1000,0,3000,0)$.

In this step, we circularly do the crossover operation process and could generate $\frac{3}{4} \times N$ new individuals.

\subsection{Mutation}

Like the crossover process, we choose several individuals to carry through mutation operation. We designate the possibility of mutation is 0.30 . Take a feasible solution for example. It is

$\mathrm{S}=(0,2000,0,4000,1000,0,3000,0)$.

We generate a random value $\theta \in(0,1)$ on every element. If $\theta \geq 0.30$, we properly adjust the possible investment on the relative element. So the feasible maybe 
$\mathrm{S}=(0,2000,0,2000,1800,0,3000,0)$.

Then we get a new population in this generation.

\section{Conclusion}

This paper describes the linguistic promotion mix management problem. Using linguistic aggregation operator $(L O W A)$, we could compute the satisfaction degree of the collective objectives in the promotion model. In order to reduce the investment account, we designate a fitness function and use another theory based on genetic algorithms to search for a better feasible solution. Finally, we could get a desirable decision model.

\section{Acknowledgement}

This work is supported by the Excellent Young Foundation of Sichuan Province (Grant No.06ZQ026-037), the National Natural Science Foundation of China (60474022), the Special Research Funding to Doctoral Subject of Higher Education Institutes in China (Grant No.20060613007) and a Project Supported by Scientific Research Fund of Sichuan Provincial Education Department(Grant No.2005A121, 2006A084).

\section{References}

[1] F. Herrera, E. Lopez and M.A. Rodriguez, A linguistic decision model for promotion mix management solved with genetic algorithms. Fuzzy Sets and Systems, 131:47-61, 2002.

[2] R.R. Yager, On ordered weighted averaging aggregation operators in multicriteria decision making. IEEE Transactions on Systems, Man, and Cybernetics, 18:183-190, 1988.

[3] Z. S. Xu, A method based on linguistic aggregation operators for group decision making with linguistic preference relations. Information Sciences, in press.

[4] F. Herrera, E. Herrera-Viedma, Linguistic decision analysis: steps for solving decision problem under linguistic information. Fuzzy Sets and Systems, 115:67-82, 2000.

[5] Z. Pei, D. Ruan, Y. Xu, J. Liu, Handling linguistic web information based on a multi-agent system. Internat. J. Intelligent Systems, 22:435453, 2007.
[6] D.E. Goldberg, Genetic Algorithms in search. Optimization, and Machine Learning (AddisonWesley, New York), 1989.

[7] Z. Miehalewicz, Genetic Algorithms + DATA Strucures $=$ Evolution Programs, Vienna, Austria, Springer-Verlag, 1996.

[8] F. Herrera, E. Lozano and J.L. Verdegay, Fuzzy connectives based crosserover operators to model gentic algorithms population diversity. Fuzzy Sets and Systems,92:21-30, 1997.

[9] J. V. Stone and J. Porrill, Undercomplete independent component analysis for signal separation and dimension reduction. Technical Report, Psychology Department, Sheffield University, Sheffield, S10 2UR, England, 1997.

[10] V. Biethahn, V. Nissen, Evolutionary Algorithms in Management Application, Springer, Berlin, 1995.

[11] F. Herrera, E. López, C. Mendaña, M. Rodríguez, Solving an assignment-selection problem with verbal information and using genetic algorithms. European J. Oper. Res, 119:326-337, 1999.

[12] F. Herrera, E. López, C. Mendaña, M. Rodríguez, A linguistic decision model for personnel management solved with a linguistic biobjective genetic algorithms. Fuzzy Sets and Systems, 118:47-64, 2001.

[13] E. López, M.A. Rodríguez, GENIA: $A$ Genetic Algorithms for Inventory Analysis. ASpreadsheet Approach, International Conference of Association for the Advancement of Modelling and Simulation Techniques in Enterprises IV, Brno, Chech Republic, pp. 200-223, 1995.

[14] E. López-Gonz ález, M.A. RodríguezFernández, C. Mendaña-Cuervo, The logistic decision making in management accounting with genetic algorithms and fuzzy sets. Mathware \& Soft Comput, 7:229-241, 2000. 